\title{
Seidel Equienergetic Graphs
}

\author{
Harishchandra S. Ramane ${ }^{1, a^{*}}$, Mahadevappa M. Gundloor ${ }^{1, b}$ \\ and Sunilkumar M. Hosamani ${ }^{2, c}$ \\ ${ }^{1}$ Department of Mathematics, Karnatak University, Dharwad - 580003, India \\ ${ }^{2}$ Department of Mathematics, Rani Channamma University, Belgaum - 591156, India \\ ahsramane@yahoo.com, bmadeshgundloor@gmail.com, csunilkumar.rcu@gmail.com \\ * Corresponding author: Harishchandra S. Ramane
}

Keywords: Seidel eigenvalues, join of graphs, Seidel energy.

\begin{abstract}
The Seidel matrix $S(G)$ of a graph $G$ is the square matrix with diagonal entries zeroes and off diagonal entries are -1 or 1 corresponding to the adjacency and non-adjacency. The Seidel energy $S E(G)$ of $G$ is defined as the sum of the absolute values of the eigenvalues of $S(G)$. Two graphs $G_{1}$ and $G_{2}$ are said to be Seidel equienergetic if $\operatorname{SE}\left(G_{1}\right)=\operatorname{SE}\left(G_{2}\right)$. We establish an expression for the characteristic polynomial of the Seidel matrix and for the Seidel energy of the join of regular graphs. Thereby construct Seidel non cospectral, Seidel equienergetic graphs on $n$ vertices, for all $n$ $\geq 12$.
\end{abstract}

\section{Introduction}

Let $G$ be a simple graph on $n$ vertices and $m$ edges with vertex set $V(G)=\left\{v_{1}, v_{2}, \ldots, v_{n}\right\}$. The Seidel matrix of a graph $G$ is an $n \times n$ real symmetric matrix $S(G)=\left[s_{i j}\right]$, where $s_{i j}=-1$ if the vertices $v_{i}$ and $v_{j}$ are adjacent, $s_{i j}=1$ if the vertices $v_{i}$ and $v_{j}$ are not adjacent and $s_{i j}=0$ if $i=j$. Obviously $S(G)=J-I-2 A$, where $J$ is the matrix whose all entries are equal to $1, I$ is an identity matrix and $A$ is the adjacency matrix of $G$. The characteristic polynomial of $S(G)$ is defined as $\not(G: \sigma)=\operatorname{det}(\sigma I-S(G))$. Let $\sigma_{1}, \sigma_{2}, \ldots, \sigma_{n}$ be the eigenvalues of $S(G)$.

The collection of the eigenvalues of the Seidel matrix of a graph is called the Seidel spectra of $G$ [6]. Two non-isomorphic graphs are said to be Seidel cospectral if their Seidel matrices have same eigenvalues.

The Seidel energy $\operatorname{SE}(G)$ of a graph $G$ is defined as [13]

$$
S E(G)=\sum_{i=1}^{n}\left|\sigma_{i}\right|
$$

The Eq. (1) is in full analogy to the ordinary graph energy defined as the sum of the absolute values of the eigenvalues of the adjacency matrix of $G$ [12]. For more details about the graph energy we refer [16]. Results on Seidel energy have been obtained in [11, 13, 18, 20].

The graphs $G_{1}$ and $G_{2}$ are said to be Seidel equienergetic if $\operatorname{SE}\left(G_{1}\right)=\operatorname{SE}\left(G_{2}\right)$. For obvious reason Seidel cospectral graphs are Seidel equienergetic. Several results on equienergetic graphs $[2,4,5$, 15, 24, 27, 28, 29, 30, 31], distance equienergetic graphs [14, 23, 26], Laplacian equienergetic graphs [8], equienergetic digraphs [17], skew equienergetic digraphs [1,25], equienergetic sigraphs $[3,9,10,19]$ and equienergetic sidigraphs $[21]$ were reported in the literature.

In [22], the Seidel energy of iterated line graphs of regular graphs was obtained and thus constructed the Seidel equienergetic graphs having different spectra. In this paper we obtain the characteristic polynomial of the Seidel matrix of the join of two regular graphs and the Seidel energy of join of regular graphs. Further we show that how to construct pairs of Seidel equienergetic graphs having different Seidel spectra, on $n$ vertices for all $n \geq 12$. 


\section{Seidel spectra and Seidel energy of join of graphs}

Definition 1: Let $G_{1}$ be the graph with vertex set $V_{1}$ and edge set $E_{1}$ and let $G_{2}$ be another graph with vertex set $V_{2}$ and edge set $E_{2}$. The union of $G_{1}$ and $G_{2}$ is the graph $G_{1} \cup G_{2}$ whose vertex set is $V_{1} \cup V_{2}$ and edge set is $E_{1} \cup E_{2}$.

Definition 2 [7]: The join of two graphs $G_{1}$ and $G_{2}$, denoted by $G_{1} \nabla G_{2}$, is a graph obtained from $G_{1} \cup G_{2}$ by joining each vertex of $G_{1}$ to all vertices of $G_{2}$.

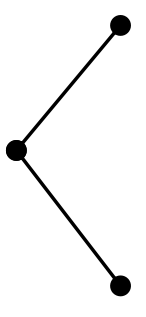

$G_{1}$

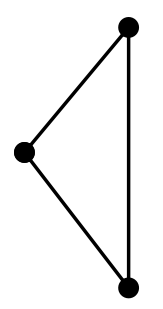

$G_{2}$

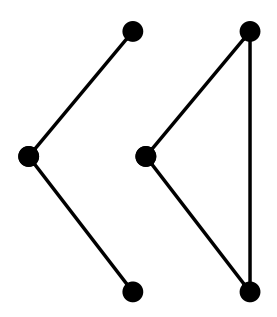

$G_{1} \cup G_{2}$

Figure 1

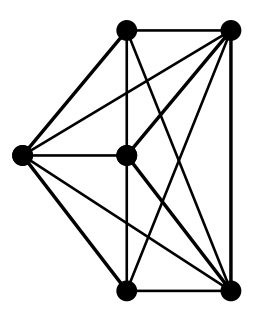

$G_{1} \nabla G_{2}$

Theorem 1: Let $G_{i}$ be an $r_{i}$ - regular graph on $n_{i}$ vertices, $i=1,2$. Then the characteristic polynomial of the Seidel matrix of $G_{1} \nabla G_{2}$ is

$$
\phi\left(G_{1} \nabla G_{2}: \sigma\right)=\frac{\left[(\sigma-X)(\sigma-Y)-n_{1} n_{2}\right]}{(\sigma-X)(\sigma-Y)} \phi\left(G_{1}: \sigma\right) \phi\left(G_{2}: \sigma\right)
$$

where $X=n_{1}-1-2 r_{1}$ and $\mathrm{Y}=n_{2}-1-2 r_{2}$.

Proof: $\phi\left(G_{1} \nabla G_{2}: \sigma\right)=\operatorname{det}\left(\sigma I-S\left(G_{1} \nabla G_{2}\right)\right)$

$$
=\left|\begin{array}{cc}
\sigma I_{n_{1}}-S\left(G_{1}\right) & -J_{n_{1} \times n_{2}} \\
-J_{n_{2} \times n_{1}} & \sigma I_{n_{2}}-S\left(G_{2}\right)
\end{array}\right|,
$$

where $J$ is a matrix whose all entries are equal to one.

The determinant (3) can be written as

$$
\left|\begin{array}{cccccccc}
\sigma & -s_{12} & \cdots & -s_{1 n_{1}} & 1 & 1 & \cdots & 1 \\
-s_{21} & \sigma & \cdots & -s_{2 n_{1}} & 1 & 1 & \cdots & 1 \\
\vdots & & \vdots & & & & \vdots & \\
-S_{n_{1} 1} & -s_{n_{1} 2} & \cdots & \sigma & 1 & 1 & \cdots & 1 \\
1 & 1 & \cdots & 1 & \sigma & -s_{12}^{\prime} & \cdots & -s^{\prime}{ }_{1 n_{2}} \\
1 & 1 & \cdots & 1 & -s_{21}^{\prime} & \sigma & \cdots & -s^{\prime}{ }_{2 n_{2}} \\
\vdots & & \vdots & & & & \vdots & \\
1 & 1 & \cdots & 1 & -s_{n_{2} 1}^{\prime} & -s_{n_{2} 2}^{\prime} & \cdots & \sigma
\end{array}\right|,
$$

where $s_{i j}$ is the $(i, j)$-th entry in $S\left(G_{1}\right), i, j=1,2, \ldots, n_{1}$ and $s_{i j}^{\prime}$ is the $(i, j)$-th entry in $S\left(G_{2}\right), i, j=1$, $2, \ldots, n_{2}$. Since $G_{i}$ is an $r_{i}$-regular graph, every vertex of $G_{i}$ is adjacent to $r_{i}$ vertices and not adjacent to $n-1-r_{i}$ vertices, $i=1,2$. Therefore

$$
\sum_{j=1}^{n_{1}} s_{i j}=n_{1}-1-2 r_{1} \text { for } i=1,2, \ldots, n_{1}
$$

and

$$
\sum_{j=1}^{n_{2}} s_{i j}^{\prime}=n_{2}-1-2 r_{2} \text { for } i=1,2, \ldots, n_{2} .
$$


We now perform a number of transformations that leave the value of the determinant (4) unchanged.

Subtract the row $\left(n_{1}+1\right)$ from the rows $\left(n_{1}+2\right),\left(n_{1}+3\right), \ldots,\left(n_{1}+n_{2}\right)$ of (4) to obtain (7):

$$
\left|\begin{array}{cccccccc}
\sigma & -s_{12} & \cdots & -s_{1 n_{1}} & 1 & 1 & \cdots & 1 \\
-s_{21} & \sigma & \cdots & -s_{2 n_{1}} & 1 & 1 & \cdots & 1 \\
\vdots & & \vdots & & & & \vdots & \\
-s_{n_{1} 1} & -s_{n_{1} 2} & \cdots & \sigma & 1 & 1 & \cdots & 1 \\
1 & 1 & \cdots & 1 & \sigma & -s_{12}^{\prime} & \cdots & -s_{1 n_{2}}^{\prime} \\
0 & 0 & \cdots & 0 & -s^{\prime}{ }_{21}-\sigma & \sigma+s_{12}^{\prime} & \cdots & -s_{2 n_{2}}^{\prime}+s^{\prime}{ }_{1 n_{2}} \\
\vdots & & \vdots & & & & \vdots & \\
0 & 0 & \cdots & 0 & -s_{n_{2} 1}^{\prime}-\sigma & -s_{n_{2} 2}^{\prime}+s_{12}^{\prime} & \cdots & \sigma+s_{1 n_{2}}^{\prime}
\end{array}\right| .
$$

Adding the columns $\left(n_{1}+2\right),\left(n_{1}+3\right), \ldots,\left(n_{1}+n_{2}\right)$ to the column $\left(n_{1}+1\right)$ of $(7)$ and using Eq. (6) we arrive at the determinant (8), where $Y=n_{2}-1-2 r_{2}$ :

$$
\left|\begin{array}{cccccccc}
\sigma & -s_{12} & \cdots & -s_{1 n_{1}} & n_{2} & 1 & \cdots & 1 \\
-s_{21} & \sigma & \cdots & -s_{2 n_{1}} & n_{2} & 1 & \cdots & 1 \\
\vdots & & \vdots & & & & \vdots & \\
-s_{n_{1} 1} & -s_{n_{1} 2} & \cdots & \sigma & n_{2} & 1 & \cdots & 1 \\
1 & 1 & \cdots & 1 & \sigma-Y & -s_{12}^{\prime} & \cdots & -s_{1 n_{2}}^{\prime} \\
0 & 0 & \cdots & 0 & 0 & \sigma+s_{12}^{\prime} & \cdots & -s_{2 n_{2}}^{\prime}+s_{1 n_{2}}^{\prime} \\
\vdots & & \vdots & & & & \vdots & \\
0 & 0 & \cdots & 0 & 0 & -s_{n_{2} 2}^{\prime}+s_{12}^{\prime} & \cdots & \sigma+s_{1 n_{2}}^{\prime}
\end{array}\right|
$$

which evidently is equal to (9):

$$
\left|\begin{array}{ccccc}
\sigma & -s_{12} & \cdots & -s_{1 n_{1}} & n_{2} \\
-s_{21} & \sigma & \cdots & -s_{2 n_{1}} & n_{2} \\
\vdots & & \vdots & & \\
-S_{n_{1} 1} & -s_{n_{1} 2} & \cdots & \sigma & n_{2} \\
1 & 1 & \cdots & 1 & \sigma-Y
\end{array}\right||B|
$$

where

$$
|B|=\left|\begin{array}{cccc}
\sigma+s_{12}^{\prime} & -s_{23}^{\prime}+s_{13}^{\prime} & \cdots & -s_{2 n_{2}}^{\prime}+s^{\prime}{ }_{1 n_{2}} \\
-s_{32}^{\prime}+s^{\prime}{ }_{12} & \sigma+s_{13}^{\prime} & \cdots & -s^{\prime}{ }_{n_{2}}+s^{\prime}{ }_{1 n_{2}} \\
\vdots & & \vdots & \\
-s_{n_{2} 2}^{\prime}+s_{12}^{\prime} & -s_{n_{2} 3}^{\prime}+s_{13}^{\prime} & \cdots & \sigma+s_{1 n_{2}}^{\prime}
\end{array}\right| .
$$

In (9) the first determinant is of order $\left(n_{1}+1\right)$. Subtract the first row from the rows $2,3, \ldots, n_{1}$, to obtain (11):

$$
\left|\begin{array}{ccccc}
\sigma & -s_{12} & \cdots & -s_{1 n_{1}} & n_{2} \\
-s_{21}-\sigma & \sigma+s_{12} & \cdots & -s_{2 n_{1}}+s_{1 n_{1}} & 0 \\
\vdots & & \vdots & & \\
-s_{n_{1} 1}-\sigma & -s_{n_{1} 2}+s_{12} & \cdots & \sigma+s_{1 n_{1}} & 0 \\
1 & 1 & \cdots & 1 & \sigma-Y
\end{array}\right||B| .
$$


Adding columns $2,3, \ldots, n_{1}$ to the first column of (11) and using Eq. (5) we get (12), where $X=$ $n_{1}-1-2 r_{1}$ :

$$
\left|\begin{array}{ccccc}
\sigma-X & -s_{12} & \cdots & -s_{1 n_{1}} & n_{2} \\
0 & \sigma+s_{12} & \cdots & -s_{2 n_{1}}+s_{1 n_{1}} & 0 \\
\vdots & & \vdots & & \\
0 & -s_{n_{1} 2}+s_{12} & \cdots & \sigma+s_{1 n_{1}} & 0 \\
n_{1} & 1 & \cdots & 1 & \sigma-Y
\end{array}\right||B|
$$

Expand it along the first column to obtain (13):

$$
\left\{(\sigma-X) \Delta_{1}+(-1)^{n_{1}} n_{1} \Delta_{2}\right\}|B|
$$

where

$$
\Delta_{1}=\left|\begin{array}{ccccc}
\sigma+s_{12} & -s_{23}+s_{13} & \cdots & -s_{2 n_{1}}+s_{1 n_{1}} & 0 \\
-s_{32}+s_{12} & \sigma+s_{13} & \cdots & -s_{3 n_{1}}+s_{1 n_{1}} & 0 \\
\vdots & & \vdots & & \\
-s_{n_{1} 2}+s_{12} & -s_{n_{1} 3}+s_{13} & \cdots & \sigma+s_{1 n_{1}} & 0 \\
1 & 1 & \cdots & 1 & \sigma-Y
\end{array}\right|
$$

and

$$
\Delta_{2}=\left|\begin{array}{ccccc}
-s_{12} & -s_{13} & \cdots & -s_{1 n_{1}} & n_{2} \\
\sigma+s_{12} & -s_{23}+s_{13} & \cdots & -s_{2 n_{1}}+s_{1 n_{1}} & 0 \\
-s_{32}+s_{12} & \sigma+s_{13} & \cdots & -s_{3 n_{1}}+s_{1 n_{1}} & 0 \\
\vdots & & \vdots & & \\
-s_{n_{1} 2}+s_{12} & -s_{n_{1} 3}+s_{13} & \cdots & \sigma+s_{1 n_{1}} & 0
\end{array}\right| .
$$

The expression (13) can be rewritten as

$$
\left\{(\sigma-X)(\sigma-Y)|A|+(-1)^{n_{1}} n_{1}(-1)^{n_{1}+1} n_{2}|A| \Delta_{2}\right\}|B|=\left\{(\sigma-X)(\sigma-Y)-n_{1} n_{2}\right\}|A||B|,
$$

where

$$
|A|=\left|\begin{array}{cccc}
\sigma+s_{12} & -s_{23}+s_{13} & \cdots & -s_{2 n_{1}}+s_{1 n_{1}} \\
-s_{32}+s_{12} & \sigma+s_{13} & \cdots & -s_{3 n_{1}}+s_{1 n_{1}} \\
\vdots & & \vdots & \\
-s_{n_{1} 2}+s_{12} & -s_{n_{1} 3}+s_{13} & \cdots & \sigma+s_{1 n_{1}}
\end{array}\right| .
$$

The determinant (15) can be written as

$$
|A|=\frac{1}{(\sigma-X)}\left|\begin{array}{ccccc}
\sigma-X & -s_{12} & -s_{13} & \cdots & -s_{1 n_{1}} \\
0 & \sigma+s_{12} & -s_{23}+s_{13} & \cdots & -s_{2 n_{1}}+s_{1 n_{1}} \\
0 & -s_{32}+s_{12} & \sigma+s_{13} & \cdots & -s_{3 n_{1}}+s_{1 n_{1}} \\
\vdots & & \vdots & \vdots & \\
0 & -s_{n_{1} 2}+s_{12} & -s_{n_{1} 3}+s_{13} & \cdots & \sigma+s_{1 n_{1}}
\end{array}\right| .
$$

From Eq. (5) the sum of the $i$-th row in (16) is $\sigma+s_{i 1}$ for $i=2,3, \ldots, n_{1}$. Therefore, by subtracting the columns $2,3, \ldots, n_{1}$ of (16) from the first column, we obtain (17):

$$
|A|=\frac{1}{(\sigma-X)}\left|\begin{array}{ccccc}
\sigma-X & -s_{12} & -s_{13} & \cdots & -s_{1 n_{1}} \\
-\sigma-s_{21} & \sigma+s_{12} & -s_{23}+s_{13} & \cdots & -s_{2 n_{1}}+s_{1 n_{1}} \\
-\sigma-s_{31} & -s_{32}+s_{12} & \sigma+s_{13} & \cdots & -s_{3 n_{1}}+s_{1 n_{1}} \\
\vdots & & \vdots & \vdots & \\
-\sigma-s_{n_{1} 1} & -s_{n_{1} 2}+s_{12} & -s_{n_{1} 3}+s_{13} & \cdots & \sigma+s_{1 n_{1}}
\end{array}\right| .
$$


Add the first row of (17) to the rows $2,3, \ldots, n_{1}$ to obtain (18):

$$
\begin{aligned}
|A|=\frac{1}{(\sigma-X)}\left|\begin{array}{ccccc}
\sigma & -s_{12} & -s_{13} & \cdots & -s_{1 n_{1}} \\
-s_{21} & \sigma & -s_{23} & \cdots & -s_{2 n_{1}} \\
-s_{31} & -s_{32} & \sigma & \cdots & -s_{3 n_{1}} \\
\vdots & & \vdots & \vdots & \\
-s_{n_{1} 1} & -s_{n_{1} 2} & -s_{n_{1} 3} & \cdots & \sigma
\end{array}\right| \\
=\frac{1}{(\sigma-X)} \phi\left(G_{1}: \sigma\right) .
\end{aligned}
$$

In a similar manner we can show that from (10) follows

$$
|B|=\frac{1}{(\sigma-Y)} \phi\left(G_{2}: \sigma\right) \text {. }
$$

Substituting (18) and (19) back into (14) gives Eq. (2).

Let $U_{1}$ and $U_{2}=V(G) \backslash U_{1}$ be the partitioned sets of the vertex set $V(G)$ of a graph $G$. Let $G^{\prime}$ be the graph obtained from $G$ by deleting all edges between $U_{1}$ and $U_{2}$ and inserting all edges between $U_{1}$ and $U_{2}$ that were not present in $G$. Then $G^{\prime}$ and $G$ are said to be Seidel switching with respect to $U_{1}$. If $G^{\prime}$ and $G$ are Seidel switching then $S\left(G^{\prime}\right)$ and $S(G)$ are similar and therefore $G^{\prime}$ and $G$ have same Seidel eigenvalues and equal Seidel energy [13]. Thus for any two graphs $G_{1}$ and $G_{2}$, the Seidel matrices $\mathrm{S}\left(G_{1} \nabla G_{2}\right)$ and $\mathrm{S}\left(G_{1} \cup G_{2}\right)$ are similar and therefore have same characteristic polynomials and $\operatorname{SE}\left(G_{1} \nabla G_{2}\right)=\operatorname{SE}\left(G_{1} \cup G_{2}\right)$.

Theorem 2: Let $G_{i}$ be an $r_{i}$-regular graph on $n_{i}$ vertices, $i=1,2$. Then

$$
S E\left(G_{1} \nabla G_{2}\right)=S E\left(G_{1}\right)+S E\left(G_{2}\right)-(X+Y)+\sqrt{(X-Y)^{2}+4 n_{1} n_{2}}
$$

where $X=n_{1}-1-2 r_{1}$ and $Y=n_{2}-1-2 r_{2}$.

Proof: From Theorem 1,

$$
\phi\left(G_{1} \nabla G_{2}: \sigma\right)=\frac{\left[(\sigma-X)(\sigma-Y)-n_{1} n_{2}\right]}{(\sigma-X)(\sigma-Y)} \phi\left(G_{1}: \sigma\right) \phi\left(G_{2}: \sigma\right)
$$

which gives that

$$
(\sigma-X)(\sigma-Y) \phi\left(G_{1} \nabla G_{2}: \sigma\right)=\left[(\sigma-X)(\sigma-Y)-n_{1} n_{2}\right] \phi\left(G_{1}: \sigma\right) \phi\left(G_{2}: \sigma\right) .
$$

Let $P_{1}(\sigma)=(\sigma-X)(\sigma-Y) \phi\left(G_{1} \nabla G_{2}: \sigma\right)$ and $P_{2}(\sigma)=\left[(\sigma-X)(\sigma-Y)-n_{1} n_{2}\right] \phi\left(G_{1}: \sigma\right) \phi\left(G_{2}: \sigma\right)$.

The roots of the equation $P_{1}(\sigma)=0$ are $X, Y$ and the eigenvalues of $S\left(G_{1} \nabla G_{2}\right)$. Therefore the sum of the absolute values of the roots of $P_{1}(\sigma)=0$ is

$$
X+Y+S E\left(G_{1} \nabla G_{2}\right) .
$$

The roots of $P_{2}(\sigma)=0$ are the eigenvalues of $S\left(G_{1}\right)$, eigenvalues of $S\left(G_{2}\right)$ and

$$
\frac{1}{2}(X+Y) \pm \sqrt{(X+Y)^{2}-4\left(X Y-n_{1} n_{2}\right)} \text {. }
$$

Therefore the sum of the absolute values of the roots of $P_{2}(\sigma)=0$ is

$$
\begin{aligned}
S E\left(G_{1}\right)+S E\left(G_{2}\right) & +\left|\frac{1}{2}(X+Y)+\sqrt{(X+Y)^{2}-4\left(X Y-n_{1} n_{2}\right)}\right| \\
& +\left|\frac{1}{2}(X+Y)-\sqrt{(X+Y)^{2}-4\left(X Y-n_{1} n_{2}\right)}\right| .
\end{aligned}
$$

Since $X Y=\left(n_{1}-1-2 r_{1}\right)\left(n_{2}-1-2 r_{2}\right)<n_{1} n_{2}$, Eq. (21) reduces to

$$
S E\left(G_{1}\right)+S E\left(G_{2}\right)+\sqrt{(X+Y)^{2}-4\left(X Y-n_{1} n_{2}\right)}=S E\left(G_{1}\right)+S E\left(G_{2}\right)+\sqrt{(X-Y)^{2}+n_{1} n_{2}} \text {. }
$$


Since $P_{1}(\sigma)=P_{2}(\sigma)$, equating Eqs. (20) and (22) we get

$$
S E\left(G_{1} \nabla G_{2}\right)=S E\left(G_{1}\right)+S E\left(G_{2}\right)-(X+Y)+\sqrt{(X-Y)^{2}+4 n_{1} n_{2}} \text {. }
$$

Let $\overline{K_{n}}$ be the totally disconnected graph on $n$ vertices and $K_{p, q}$ be the complete bipartite graph on $p+q$ vertices.

Corollary 2.1: $S E\left(K_{p, q}\right)=S E\left(\overline{K_{p}} \nabla \overline{K_{q}}\right)=2(p+q-1)$

Corollary 2.2: If $H_{1}$ and $H_{2}$ are Seidel non cospectral, Seidel equienergetic regular graphs on $n$ vertices and of same degree, then for any regular graph $G, S E\left(H_{1} \nabla G\right)=S E\left(H_{2} \nabla G\right)$.

\section{Construction of Seidel equienergetic graphs}

Consider the graphs $H_{a}$ and $H_{b}$ as shown in Fig. 2.
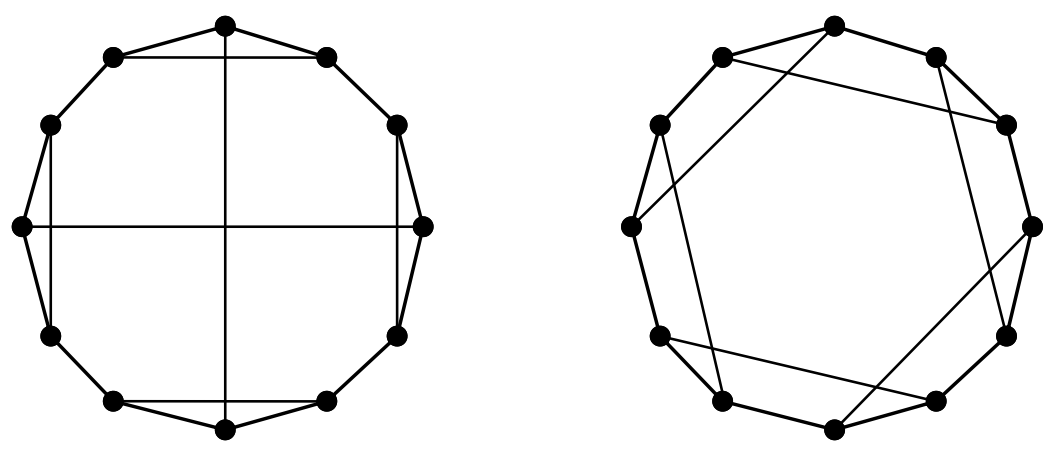

$H_{a}$

Figure 2

By direct computation,

$$
\phi\left(H_{a}: \sigma\right)=(\sigma-5)(\sigma-3)^{3}(\sigma-1)^{3}(\sigma+1)^{2}(\sigma+5)^{3}
$$

and

$$
\phi\left(H_{b}: \sigma\right)=(\sigma-5)^{2}(\sigma-3)^{2}(\sigma-1)(\sigma+1)^{4}(\sigma+3)(\sigma+5)^{2} .
$$

Both $H_{a}$ and $H_{b}$ are regular graphs on 12 vertices and of degree 3. And $S E\left(H_{a}\right)=S E\left(H_{b}\right)=34$. Let $H$ be any $r$-regular graph on $p \geq 1$ vertices. Then by Theorem 2 ,

$$
S E\left(H_{a} \nabla H\right)=S E\left(H_{b} \nabla H\right)=S E(H)+30-p+2 r+\sqrt{(6-p+2 r)^{2}+48 p} .
$$

Thus $H_{a} \nabla H$ and $H_{b} \nabla H$ are Seidel equienergetic. By Eqs. (23) and (24), $H_{a}$ and $H_{b}$ are Seidel non cospectral, so by Theorem $1, H_{a} \nabla H$ and $H_{b} \nabla H$ are also Seidel non cospectral. Further $H_{a} \nabla H$ and $H_{b} \nabla H$ possesses equal number of vertices $n=12+p, p=1,2, \ldots$.

Also for $n=12$, the Seidel equienergicity is directly verified from Eqs. (23) and (24).

From above construction, we state the following Theorem.

Theorem 3: There exist pairs of Seidel non cospectral, Seidel equienergetic graphs on $n$ vertices, for all $n \geq 12$.

Let $K_{p}$ be the complete graph on $p$ vertices. It is regular of degree $p-1$. The Seidel matrix of $K_{p}$ is the negation of its adjacency matrix. Therefore $S E\left(K_{p}\right)=2(p-1)$ [7]. Using this in Theorem 2 we have following result.

Theorem 4: If $H_{a}$ and $H_{b}$ are the graphs as shown in Fig. 2, then for $p \geq 1$,

$$
S E\left(H_{a} \nabla K_{p}\right)=S E\left(H_{b} \nabla K_{p}\right)=3 p+26+\sqrt{p^{2}+56 p+16} .
$$




\section{Conclusion}

From Corollary 2.2, the construction of Seidel non cospectral, Seidel equienergetic graphs on equal number of vertices can be done. In particular from Theorem 3 and Theorem 4, it is easy to construct a pair of Seidel non cospectral, Seidel equienergetic $n$-vertex graphs for all $n \geq 12$.

\section{Acknowledgement}

The author HSR is thankful to University Grants Commission (UGC), Govt. of India for support through research grant under UPE FAR-II grant No. F 14-3/2012 (NS/PE). Another author MMG is thankful to the Karnatak University for support through UGC-UPE scholarship No. KU/Sch/UGCUPE/2013-14/1136.

\section{References}

[1] C. Adiga, R. Balakrishnan, W. So, The skew energy of digraphs, Linear Algebra Appl. 432 (2010) 1825-1835.

[2] R. Balakrishnan, The energy of a graph, Linear Algebra Appl. 387 (2004) 287-295.

[3] M. A. Bhat, S. Pirzada, On equienergetic signed graphs, Discrete Appl. Math. 189 (2015) 17.

[4] A. S. Bonifacio, C. T. M. Vinagre, N. M. M. de Abreu, Constructing pairs of equienergetic and non-cospectral graphs, Appl. Math. Lett. 21 (2008) 338-341.

[5] V. Brankov, D. Stevanovic, I. Gutman, Equienergetic chemical trees, J. Serb. Chem. Soc. 69 (2004) 549-553.

[6] A. E. Brouwer, W. H. Haemers, Spectra of Graphs, Springer, Berlin, 2012.

[7] D. M. Cvetkovic, M. Doob, H. Sachs, Spectra of Graphs, Academic Press, New York, 1980.

[8] H. A. Ganie, S. Pirzada, A. Ivanyi, Energy, Laplacian energy of double graphs and new families of equienergetic graphs, Acta Univ. Sapientiae Info. 6 (2014) 89-116.

[9] K. A. Germina, K. S. Hameed, On signed paths, signed cycles and their energies, Appl. Math. Sci. 4 (2010) 3455-3466.

[10] K. A. Germina, K. S. Hameed, T. Zaslavsky, On product and line graphs of signed graphs, their eigenvalues and energy, Linear Algebra Appl. 435 (2011) 2432-2450.

[11] E. Ghorbani, On eigenvalues of Seidel matrices and Haemers conjecture, arXiv: $1301.0075 \mathrm{v} 1$.

[12] I. Gutman, The energy of a graph, Ber. Math. Stat. Sekt. Forschungsz. Graz 103 (1978) 1-22.

[13] W. H. Haemers, Seidel switching and graph energy, MATCH Commun. Math. Comput. Chem. 68 (2012) 653-659.

[14] G. Indulal, I. Gutman, A. Vijaykumar, On the distance energy of a graph, MATCH Commun. Math. Comput. Chem. 60 (2008) 473-484.

[15] G. Indulal, A. Vijaykumar, Equienergetic self-complementary graphs, Czechoslovak Math. J. 58 (2008) 451-461.

[16] X. Li, Y. Shi, I. Gutman, Graph Energy, Springer, New York, 2012.

[17] W. Lopez, J. Rada, Equienergetic digraphs, Int. J. Pure Appl. Math. 36 (2007) 361-372.

[18] Nageswari, P. B. Sarasija, Seidel energy and its bounds, Int. J. Math. Analysis 8 (2014) 2869-2871.

[19] N. G. Nayak, Equienergetic net-regular signed graphs, Int. J. Contemp. Math. 9 (2014) 685693.

[20] M. R. Oboudi, Energy and Seidel energy of graphs, MATCH Commun. Math. Comput. Chem. 75 (2016) 291-303.

[21] S. Pirzada, M. A. Bhat, Energy of signed digraphs, Discrete Appl. Math. 169 (2014) 195205. 
[22] H. S. Ramane, I. Gutman, M. M. Gundloor, Seidel energy of iterated line graphs of regular graphs, Kragujevac J. Math. 39 (2015) 7-12.

[23] H. S. Ramane, I. Gutman, D. S. Revankar, Distance equienergetic graphs, MATCH Commun. Math. Comput. Chem. 60 (2008) 473-484.

[24] H. S. Ramane, I. Gutman, H. B. Walikar, S. B. Halkarni, Equienergetic complement graphs, Kragujevac J. Sci. 27 (2005) 67-74.

[25] H. S. Ramane, K. C. Nandeesh, I. Gutman, X. Li, Skew equienergetic digraphs, Trans. Comb. 5 (2016) 15-23.

[26] H. S. Ramane, D. S. Revankar, I. Gutman, H. B. Walikar, Distance spectra and distance energies of iterated line graphs of regular graphs, Publ. Inst. Math. (Beograd) 85 (2009) 3946.

[27] H. S. Ramane, H. B. Walikar, Construction of equienergetic graphs, MATCH Commun. Math. Comput. Chem. 57 (2007) 203-210.

[28] H. S. Ramane, H. B. Walikar, S. B. Rao, B. D. Acharya, P. R. Hampiholi, S. R. Jog, I. Gutman, Equienergetic graphs, Kragujevac J. Math. 26 (2004) 5-13.

[29] H. S. Ramane, H. B. Walikar, S. B. Rao, B. D. Acharya, P. R. Hampiholi, S. R. Jog, I. Gutman, Spectra and energies of iterated line graphs of regular graphs, Appl. Math. Lett. 18 (2005) 679-682.

[30] D. Stevanovic, Energy and NEPS of graphs, Lin. Multilin. Algebra 53 (2005) 67-74.

[31] L. Xu, Y. Hou, Equienergetic bipartite graphs, MATCH Commun. Math. Comput. Chem. 57 (2007) 363-370. 